\title{
Welcome Message from DSE Managing Editor
}

\author{
Lizhu Zhou ${ }^{1}$
}

Published online: 1 March 2016

(C) The Author(s) 2016. This article is published with open access at Springerlink.com

Welcome to the Data Science and Engineering (DSE) journal, an academic platform for sharing your achievements in data science and related research with scientists and engineers across the world.

In recent years, the Chinese database community, represented by the China Computer Federation (CCF) Technical Committee on Databases (TCDB), has contributed a substantial number of publications to top conferences, including VLDB, SIGMOD, ICDE, WWW, and KDD. At the same time, collaboration between Chinese institutions and international partners in organizing international conferences and performing joint research has increased considerably. These are dramatic changes compared with the database research activities of the early years of this century. Similar trends have been observed within other technical committees of CCF, such as those focusing on the Internet, High-Performance Computing, and the Internet of Things. This progress in database research in China is continually being pushed forward by the worldwide development of the area of big data technologies and applications. Inspired by these exciting trends, we decided to develop a new journal so that scientists and engineers both inside and outside of China could share research results on the foundations and novel applications of big data technology. With this aim in mind, we formed

On behalf of TCDB of CCF.

Lizhu Zhou

dcszlz@mail.tsinghua.edu.cn

1 Beijing, China a special group within TCDB focusing on this project. In January of 2015, we achieved a milestone; a formal agreement on establishing a new journal-titled Data Science and Engineering - was signed between CCF, Springer, and Nanjing Sinovatio Tech. Co. Ltd. In the summer of 2015, the DSE Web site went live and started accepting submissions. DSE has now become an official publication of $\mathrm{CCF}$, sponsored by TCDB.

DSE is an international journal. Members of the editorial board, led by the Editors-in-Chief Professor Jianzhong Li from Harbin Institute of Technology and Professor Elisa Bertino from Purdue University, have a strong background in databases, data mining and analytics, privacy and security, machine learning, etc., and are based in different countries throughout the world. The advisory board of DSE consists of well-known experts from seven different countries. DSE applies an open access publication model, and the authors publish free of charge. The publication fees are covered by sponsorship. Papers submitted to DSE are subject to strict peer review and published on the Web as soon as they have been approved by the Editors-in-Chief. All of these measures ensure that DSE is an ideal and efficient platform for you to submit papers reporting the latest output of your research in a timely way. Here, through this welcome message, we cordially invite you to submit to the DSE journal.

We would like to thank the Editors-in-Chief, Professor Elisa Bertino and Professor Jianzhong Li, for devoting their invaluable time and effort to DSE. We would like to thank Alfred Hofmann, Springer Vice President Computer Science Publishing, and Celine Chang, Senior Editor of Springer Beijing Office, for their supportive cooperation. We are grateful 
to Nanjing Sinovatio Tech. Co. Ltd. for sponsoring the open access fees for all DSE papers. Last but not least, we would like to thank those colleagues of TCDB who have devoted their time and energy to the setup of DSE. It would be very hard to imagine the establishment of DSE without your help.
Open Access This article is distributed under the terms of the Creative Commons Attribution 4.0 International License (http://creativecomm ons.org/licenses/by/4.0/), which permits unrestricted use, distribution, and reproduction in any medium, provided you give appropriate credit to the original author(s) and the source, provide a link to the Creative Commons license, and indicate if changes were made. 